\title{
Design and Application of the Intelligent Operation, Control and Maintenance of Distribution Networks Based on Wide-Area Distributed Sinking Computing Technology
}

\author{
Xiangjun DUAN, Jia LI ${ }^{1}$ and Dezhi FENG \\ China Electric Power Research Institute, Beijing 100192, China
}

\begin{abstract}
The distribution network (DN) is characterized by wide coverage and highly scattered data distribution. To improve the business process and data calculation capability for DN management, a foundational computing technology, the wide-area distributed sinking calculation technology (W-A DSCT ), is proposed based on the application of big data, artificial intelligence, block chain and other technologies. It is applicable to the OCMS (operation, control and maintenance) of distribution networks. The technology adopts the concept of "moving computation, not moving to data" to realize fast processing of business applications. The algorithm and business logic are designed at the primary node level, and the algorithm compilation and data crawling and mining are performed at the sub-node level to realize the fast business response. This paper introduces the technical implementation methods and technologies of each link, and performs a comparative analysis for typical business scenarios between the sinking calculation scheme and the original framework calculation. It is verified that applying the sinking calculation scheme can significantly enhance the rapid processing ability and computational efficiency.
\end{abstract}

Keywords. Distribution Network, Sinking Calculation, Big Data, Artificial Intelligence, Block Chain.

Abbreviations
\begin{tabular}{|ll|}
\hline DN & Distribution Network \\
W-A DSCT & Wide-Area Distributed Sinking Calculation Technology \\
AI & Artificial Intelligence \\
DPCT & Distributed Parallel Computing Technology \\
IoT & Internet of Things \\
PSSC & Power Supply Service and Command \\
OCMS & Operation, Control and Maintenance System \\
PMS & Power Production Management System \\
OMS & Outage Management System \\
\hline
\end{tabular}

1 Corresponding Author, Jia LI, China Electric Power Research Institute, China; E-mail: 1115273450@qq.com. 


\section{Introduction}

With the rapid development of the society and economy, the electricity demand continues to increase, the scale of DN keeps expanding and the number of power users keeps growing, which leads to the increment of the data scale. The continuous upgrading of the Internet technology has pushed the information society into the era of big data[1], and the AI technology stems from big data[2].

In China, the PSSC platform makes full use of the professional information of the dispatching, operation, maintenance and marketing systems, and integrates the functions of the marketing system, PMS system, real-time production control, electricity acquisition, scheduling automation, distribution automation, OMS and other system. The main functions of the regional management are designed and developed such as the emergency repair management, marketing work order management, hotline application for work order, supervisory work order management, active service management, power outage information release and etc[3]. The construction and improvement of the platform have promoted the improvement of the management ability of the DN[4]. However, due to the huge scale of DN nodes, the application of power flow calculation and state estimation in distribution automation system is often accompanied by a large number of matrix operation and loop iteration. Due to the restriction of data magnitude, the analysis and decision are directly affected, and the calculation speed is difficult to meet the requirements.

Taking the State Grid Corporation of China as an example. Although each provincial company has a big data center, which is capable of massive data storage and simple queries, the independent databases and lack of distributed processing technology deployment make it impossible to realize the rapid processing of the highly diversified data and deep mining[5] of the potential value to support the management decision analysis. The DPCT has the characteristics of high-speed data exchange, which can realize the data acquisition and distributed storage[6]. This technology can be used for the hierarchical deployment of data processing to meet the needs of the centralized control and hierarchical governance of the production and operation management for the DN. The DN data is getting bigger, it is urgent to use the DPCT (distributed parallel computing technology) to improve the computing power of data[7]. The computing tasks are first segmented and then integrated, which can meet the needs of DPCT and ensure the good interaction of the system.

Based on the characteristics of data structure, a W-A DSCT is proposed in this paper. The special power grid business algorithm and general statistical analysis algorithm are adopted and the AI, containerization, edge computing and the block chain technology are applied for the deep mining of data[8], building the multi-scenarios[9] and effectively penetrating the data step by step. The core technology of the ubiquitous IoT[10] platform can be established to realize the in-depth analysis of multi-dimensional data providing the intelligent management and decision support and effectively improving the lean management of the DN. 


\section{Framework Design}

\subsection{Overall Framework}

Under the current information structure of the DN, the intelligent OCMS of the DN is designed based on the provincial PSSC platform and W-A DSCT to make the information business of the DN online, transparent, mobile and intelligent. Then a customer-centered DN operation mechanism is established to promote the operation concept, to further improve the efficiency and benefit of the DN operation achieving the interconnection with the provincial PSSC platform, the penetration, control and in-depth analysis of data.

The intelligent OCMS of the DN is connected with the PSSC platform, and the big data computing resources, storage resources and data resources of the big data platform are used to support the construction of the center operation and control platform. The total node in the system has the functions of graphical modeling, visual design, algorithm issuing and management, etc. The sub-node is the sinking calculation node. It mainly carries out the sinking algorithm and business description, carries out the unified scheduling through computing the service resources of provincial nodes. And the subnode connects with the PSSC platform, it calculates and analyzes the data based on computing resources of the big data platform. The computing results are returned to the center operation and control platform, as shown in figure.1.

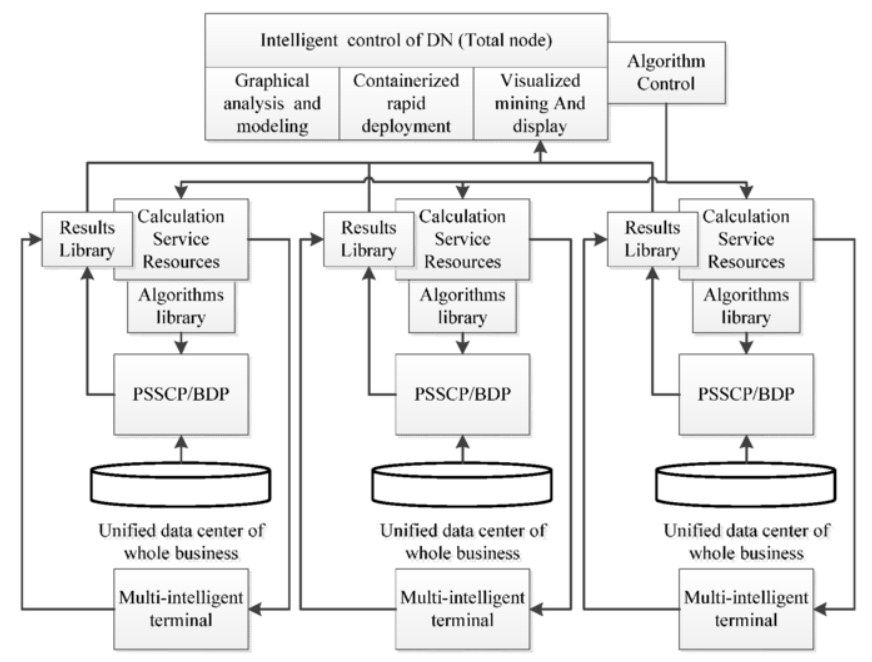

Figure 1. Logical framework of this paper

\subsection{Logical Framework}

The W-A DSCT logically adopts multi-layer application technology. As shown in figure 2 , the total node takes the basic cloud platform as the infrastructure support, and takes the container cloud platform as the carrier of services and applications, and the Hadoop cloud platform as the carrier of big data storage and analysis to provide the upper layer with big data storage, offline analysis, micro-computing, micro-service and other application functions. 
It can realize the self-service business scenario analysis such as the intelligent DN operations management, a panoramic view of virtual reality modeling visualization and graphical data mining algorithm based on the storage, calculation, analysis, data mining, and other functions of the underlying cloud platform and wide-area distributed platform combined with the business needs of the DN operation.

The sub-nodes include the analysis computing nodes, all business data center and wide-area distributed nodes of big data platform. The total node distributes the computing tasks to the sub-node through the network channel. Through the sub-nodes, the data interaction of the business data center and big data platform can be realized to carry out the calculation and analysis of the data in PSSC, and finally the calculation results would be uploaded to the total nodes, and the results can be analyzed, summarized and shown by the total nodes.

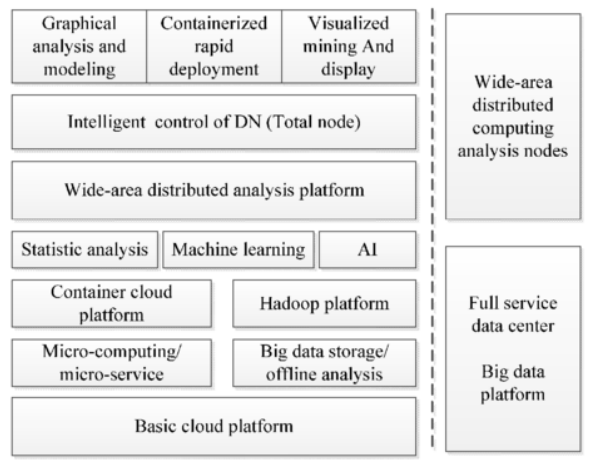

Figure 2. Logical framework

\section{Key Technologies}

\subsection{W-A DSCT Technology}

In order to realize the requirement of "moving computation, not moving to data", a fourlevel analytical computing system is designed, which is the algorithm layer, the analysis layer, the fusion layer and the calculation layer. In the algorithm layer, a progressive and syncretic sinking algorithm model is designed containing operators and examples.

(1) Distributed scheduling of algorithm load

After the business logic algorithm is decomposed by the total node, the sub-node services are distributed on a large scale through MapReduce, and the computing logic and business requirements of the node are sorted out. Data sets and data sources are distributed on a large scale to ensure the reliability of the algorithm in distributed scheduling applications. Each node periodically returns the completed work and updates the latest status to confirm the existence of the node. The computing tasks are divided according to the requirements of the unity algorithm, and the computing tasks of the subnodes are unified and balanced. However, since the intermediate results are allocated to the same Reducer, which are output by the Mapper with the same hash key value for processing in the Reduce stage. The uneven distribution of data results in different tuples number per key, data skew occurs, and the amount of data received by Reducer is unbalanced. If the amount of data is too large, the node operation speed will slow down, 
which will seriously restrict the completion speed of the whole task. Therefore, after the hash distribution, the normal distribution calculation is carried out according to the calculation business logic and the calculation demand of node resources in the MAP stage of the algorithm. The second-order curve of business volume and calculation quantity is established. According to the dynamic distribution, the task logic of the modified algorithm is distributed, so as to realize the dynamic distribution of the algorithm in each sub-node. The dynamic distributed scheduling of the algorithm load is realized by modifying the calculation based on the feedback of nodes.

(2) AI analysis preloading

In the process of users' response, the analysis results requires deep mining to ensure the effective analysis results. Inputting the AI analysis logic formula, the memory dynamic allocation mechanism is established to carry out the data calculation in blocks, progressive mining, repetitive matching, and storage of the calculation logic process in the cache improving the response speed of the calculation process.

In order to realize the preloading of AI analysis, the structure sets multiple partitions, and the AI algorithm is embedded on the independent associated partition block, and the fuzzy matching sequence is established for the algorithm input and added to the cache. The block cache is preloaded, that is, the cache of the next item is preloaded before the application computation request has read the last item in the block. This loading method can improve the calculation speed and response efficiency, reduce the read times of DB in the process of distributed data response and lower the size of data transmitted in the cache avoiding the request timeouts for block-caching applications that are preloaded in response to computational requirements.

(3) Containerization and rapid sinking

The Docker Engine loaded by the sinking computing nodes can be used as a daemon on the two-level nodes, providing services such as rapid delivery of business applications based on the specific requirements of the total node design, dynamic management, allocation and real-time deployment of the sinking application service resources.

\begin{tabular}{|c|c|c|c|c|c|}
\hline \multicolumn{3}{|c|}{ Application } & \multicolumn{3}{|c|}{ Algorithm } \\
\hline $\begin{array}{l}\text { Application } \\
\text { No.1 }\end{array}$ & $\begin{array}{l}\text { Application } \\
\text { No. } 2\end{array}$ & $\begin{array}{l}\text { Application } \\
\text { No. } 3\end{array}$ & $\begin{array}{c}\text { Algorithm } \\
\text { No.1 }\end{array}$ & $\begin{array}{c}\text { Algorithm } \\
\text { No.2 }\end{array}$ & $\begin{array}{c}\text { Algorithm } \\
\text { No.3 }\end{array}$ \\
\hline \multicolumn{3}{|c|}{ Operating environment } & \multicolumn{3}{|c|}{ Operating environment } \\
\hline \multicolumn{6}{|c|}{ Docker container engine } \\
\hline \multicolumn{6}{|c|}{ Host operating system } \\
\hline \multicolumn{6}{|c|}{ Hardware layer } \\
\hline
\end{tabular}

Figure 3. W-A DSCT Technology

The main principle of sinking calculation (figure 3):

- construct the application image by sending the primary node through the sinking channel, run the image on the sub-node to form a relatively independent application container and complete the specific business requirements calculation and analysis tasks; 
- update the corresponding calculation and analysis method according to the changing business requirements by the container and repack and send to the sub-node for computation.

In the container resource management, the container engine mainly realizes the isolation control of the container resource through Cgroup technology to perform corresponding Linux process of the container. The Cgroup can limit the amount of resources used by a process group, including CPU, memory, disk, network bandwidth, and so on. In addition, Cgroup can also set the priority of the process, suspend the process, resume and perform other operations.

\subsection{LAN Distributed Computing Technology}

After receiving the demand of the primary node, the secondary distribution of MapReduce is conducted. Analyzing and scheduling the resources of the same cluster in the node environment, it can be realized that the distributed computing of data. The computing process is connected with the resources of the big data platform to realize the computing of the business. The data source is sunk, the index and instructions are sent to the acquisition terminal through the network, and the terminal performs calculation after a data collection.

The sub-nodes are connected with the provincial big data centers and full-business data centers. Through Spark technology as shown in figure 4, the unified cross-domain distribution application of data resources can be realized, interactive iteration is established, iteration workload can be optimized, and the algorithm and business are integrated.

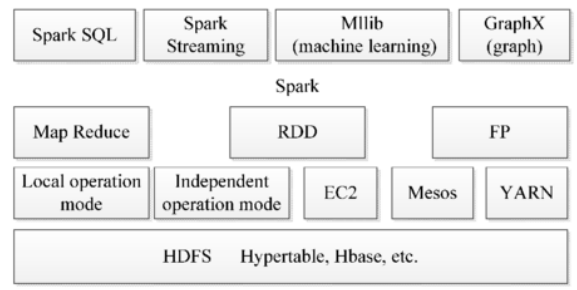

Figure 4. Application of Spark

Spark does not include the distributed file system. The application of node resources mainly relies on HDFS storage resources in the provincial big data and full-business data centers, which are utilized for the computing. After obtaining the requirements of the computing task, they were decomposed. And the requirements were deeply filtered through Stage 1. After the analysis is called, Stage 2 is carried out to distribute the overall and normalized work tasks. Then the decomposition factors are obtained through the corresponding relationship between Stage 3 and the business of the full-business data center. 


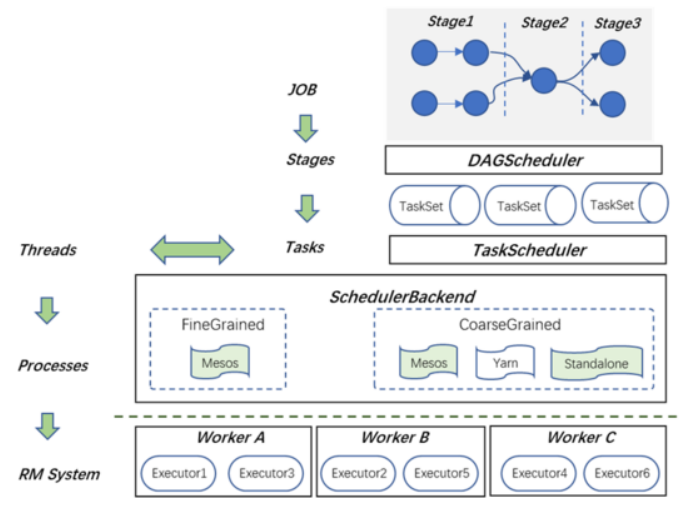

Figure 5. The procession of the DAG scheduler

Through DAG scheduler (figure 5), the available task set containing the application of algorithm is established according to the dependency and data of computing tasks, and the task is decomposed through the task management and scheduler. The independent and non-independent relational classification process is established for mining and learning, and then the analysis is carried out through the different task actuators.

Since the data resources and sub-nodes belong to the provincial company layer, the data calculation constructed by this layer is defined as local calculation. At the computing level, artificial intelligence algorithm is applied to realize machine learning on the basis of computing and to carry out deep mining of business requirements.

\subsection{Terminal Distributed Edge Computing}

Distribution terminal is the base of DN data source, which has a large number of basic operation data. At the center level, the intelligent distribution terminal has the function of edge computing, which mainly completes the basic analysis and calculation of fixed applications and builds a channel for professional and directional data analysis. As the collection point of data sources, distribution terminal cannot fully utilize the data and computing resources. Therefore, the application side of terminal computing is constructed through the W-A DSCT, and the data silos between business and data is broken through by using the physical sinking channel, so as to realize direct service and data connection.

The edge computing technology is applied for data calculation and analysis in the most marginal area of data, so as to get close to users and networked devices and respond to the needs of computing processing more conveniently and quickly.

All intelligent terminals with edge computing capabilities are integrated into the edge computing nodes of W-A DSCT. Block chain technology is applied to satisfy the distributed requirements of business algorithms in terminal computing.

\section{Intelligent scheduling of terminal computing resources:}

Block chain technology is a new application model of advanced technologies such as distributed data storage, point-to-point transmission, consensus mechanism and encryption algorithm. And it has the characteristics of subject equivalence, openness and transparency, secure communication, tamper-proof and multi-party consensus, it will have an important impact on the IoT. The characteristics of multi-center and weakly centralized architecture will reduce the high operation and maintenance costs of the centralized architecture. Information encryption and secure communication is crucial to 
protect privacy, identity manage and multi-party consensus, and identify illegal nodes, so as to timely prevent accessing and attacking of malicious nodes.

At present, according to its own business data attributes, intelligent terminals rely on the chain structure to build authenticated and traceable data source electronic storage certificate. The characteristics of distributed architecture and subject equivalence can solve the problem of multiple information islands in the IoT, which enables the edge of the current intelligent terminal with data flow and multi-party cooperative computing capabilities. In W-A DSCT, the intelligent IoT terminal is used as the computing nodes of the main block chain. Through business attribute and the information carried by the intelligent terminals, it is shown that each intelligent terminal data has the unique and special characteristics, the free scheduling of computing resource for terminal can be realized, the efficiency of terminal resources calculation, business matching accuracy and uniqueness can be improved.

The nodes of W-A DSCT are taken as the center to distribute the business requirements. And each IoT node of the distributed terminal is taken as the terminal of distributed computing to realize the computing function. When computing resources required by nodes are issued, the block chain technology is used to dynamically allocate terminal computing resources and conduct distributed edge computation.

\section{Fast Response of Edge Calculation:}

Automatic intelligent terminal, "control" is regarded as the core function. The control function is based on "signal", and "calculation" is based on data. For business data, it refers to "strategy" and "planning". Therefore, edge computing focuses on "scheduling and optimization". If multiple nodes collect data at the same time, the sudden increase of load is bound to adjust the operation mode of distribution transformer accordingly. Based on the operation and planning of time and nodes, intelligent terminals are used to carry out edge calculation of the IoT, and control and calculation requirements can be quickly responded based on the information. Each terminal is used as the data source to construct the final IoT nodes of W-A DSCT.

\subsection{Algorithm Encapsulation and Big Data Resource Sharing}

The core of sinking calculation is to subcontract and encapsulate the algorithm and establish the logical relationship of the algorithm with the operators as the calculation molecule. Endowing the business logic to the algorithm, and three kinds of calculation rules are established according to the requirements of business computing: big data mining algorithm, business proprietary algorithm and general logic algorithm. The three algorithms are put into the corresponding calculation rules respectively for operator sinking. On the sinking calculation node, the algorithm is compiled to establish the corresponding relationship between requirements and data, and the algorithm decomposition is realized (operators are determined according to requirements, and operators can also constitute the independent algorithms).

There are differences in data specifications of full-service data centers, including structured data in relational databases, semi-structured and unstructured data stored in XML, Excel and other files. The complexity of data makes it difficult to share data among different systems. 


\section{Intelligent Design of the Management and Maintenance for the DN}

The basic requirements of the DN are to realize the continuity and reliability of power supply, to ensure power quality and to meet the economic requirements of operation. And also the DN shall have the necessary capacity margin, ability of appropriate load transfer, certain self-healing and emergency handling ability, and reasonable hosting capacity of distributed energy resources. With the PSSC platform as the foundation support, the platform application system is designed integrating professional business and oriented business of the sinking management.

\subsection{Service Regulation of $D N$}

The supervision level can realize the analysis and display of key operating indicators of equipment, engineering and operation quality. And it can regulate the reasonable intervals of various indicators, track the trend of indicators, verify the effectiveness of business development, and assist in finding weak indicators.

\subsection{Optimization of Power Supply Service}

Applying the results of the deep integration between the operation, distribution and adjustment, it can be realized that the quick acquisition, accurate research and judgment, accurate positioning and resource allocation combined with the grid topology, two-way verification and efficient command of emergency repair work. It will change the passive rush repair to active service, improve the efficiency and quality service level.

\subsection{Command Service Management}

Based on extensive application in electric power IoT, data mining, Internet technology, and GIS geographical information technology, the DN fault analysis and visual positioning can be realized. It can support the production, emergency maintenance and customer service of DN. Active repair, overhaul and service can be realized, the working efficiency of emergency repair and the quality of power supply service will be improved.

\section{Design of the Core Function Management}

\subsection{Graphical Algorithm Modeler}

The graphical algorithm modeler is the core function of the system. Based on the data model provided by the big data resource manager and the algorithm operator model provided by the algorithm operator manager, the algorithm model of sinking calculation is graphitized, and the algorithm model is encapsulated and sinking by means of dragging and dropping.

(1) Basic operation

The graphical algorithm modeler supports the infinite vector scaling of the graph, the undo and redo of various operations, the flexible drag and drop of the data model and the algorithm operator model, the copy, paste and delete of the model primitives, and the connection between the model primitives. 
(2) Algorithm model construction

According to the business requirements, the required data model and algorithm can be dragged and dropped to graphical operator model algorithm modeler. The corresponding parameter information is configured respectively and the algorithm model is established. All kinds of operators are connected by the lines with arrows to reflect their relationship of input and output. The end of the arrow is the input model, and the front of the arrow is the operator model that receives the input. The direction of the arrow indicates the flow direction of the data.

(3) Encapsulation and sinking of algorithm model

After the construction of the algorithm model, the corresponding sinking calculation nodes can be selected to carry out the sinking of the algorithm. Before sinking, the algorithm model will be encapsulated, the data model and the algorithm operator model will be extracted. According to the selected sinking calculation nodes, the parameters are adapted and the algorithm model was re-encapsulated.

\subsection{Big Data Manager}

The big data resource manager can realize the access management and monitoring of the shared business data resources of the big data platform for the sinking computing node to provide the data model for graphical modeling.

(1) Access management of data resource

Access management of data resource means that it maintains the access to business data resources that can be shared by the big data platform of sinking computing nodes, including the name, description, storage location, domain, column information of the data resources, etc. Meanwhile, it can monitor the status of data resources for each sinking computing nodes.

(2) Data resource information monitoring

The basic information of data resources for each sinking calculation node is monitored, including the size, volume and last modification time, so as to timely detect abnormal situations and to ensure the timeliness of data resources and the accuracy of sinking calculation.

(3) Data resource information statistics

The basic information of the data resources for each sinking calculation node was analyzed by multi-dimensional statistics. For the data dimension, the size, volume and other aspects of data resources can be analyzed. For the time dimension, the basic information of data resources can be analyzed by day, month, season and year.

(4)Acquisition of data resource samples

The sample data of the specified data resources can be obtained by selecting the sinking calculation nodes to conduct the business demand analysis.

(5) Overview of data resources

According to the data domain, it provides the retrieval overview function of data source, supports the drag and drop and other functions, realizes the encapsulation of data resources, and provides the data model for the graphical algorithm modeler.

\subsection{Algorithm Operator Manager}

The algorithm operator manager encapsulates all kinds of algorithm operators and provides algorithm operator models for graphical algorithm modeler. The algorithm 
model mainly includes classification algorithm, clustering algorithm, regression algorithm, recommendation algorithm and so on.

(1) Classification algorithm

The classification algorithm is a supervised learning algorithm, which can obtain the mapping between the sample features and the sample labels according to the training samples, and the mapping can be used to obtain the new sample labels and to classify the new samples into different categories. Classification algorithms mainly include Bayesian classification, SVM (support vector machine), decision tree and so on.

(2) Clustering algorithm

Clustering algorithm is a typical unsupervised learning algorithm, and it is a kind of algorithm to automatically classify things. In the clustering algorithm, things with similar attributes are classified into the same category by defining different similarity measurement methods. Clustering algorithms mainly include K-means, LDA (Linear Discriminant Analysis) topic model algorithm, etc.

(3) Regression algorithm

Regression algorithm is a supervised learning algorithm. The sample labels are continuous values, and the mapping between sample features and continuous labels is obtained through training.

(4) Recommendation algorithm

Recommendation algorithm is to surmise users' preferences based on users behavior through mathematical algorithm. Recommendation algorithms mainly include ALS (Alternating Least Square) and collaborative filtering recommendation algorithm.

\section{Scene Case}

A scenario of the W-A DSCT is constructed which include the two-level calculation nodes. The average power return time (monthly) index was taken as an example for calculation and analysis. Among them, the management node is equipped with six cloud virtual hosts, eight-core CPU, 64GB memory, 2BT storage; computing sub-nodes use eight cloud virtual hosts with the same configuration as the management node. The management node is connected with the computing sub-node through Ethernet, and its internal servers are connected through the Gigabit network. Hadoop2.6.0, Spark1.6.0 and other components are used as the distributed computing platform.

\subsection{Rapid Mining and Calculation Analysis of the Business Indicators}

A total of 21,104 pieces of work order samples were collected. Define a database to record transactions, represented as table 1:

Table 1. Transactions database

\begin{tabular}{ll}
\hline TID & \multicolumn{1}{c}{ Items } \\
\hline T1 & Troubleshooting time, processing site classification, on-duty time \\
T2 & Weather, location \\
T3 & First class classification, second class classification \\
T4 & Device attributes, level, classification \\
T5 & Arriving time, type of treatment \\
T6 & Fault classification, troubleshooting time \\
\hline
\end{tabular}


Comparing the correlation degree and letting MinSupport $=6$ (the minimum correlation degree), the one-dimensional maximum project set (L1) is generated, shown in table 2 .

Table 2. One-dimensional maximum project set L1

\begin{tabular}{lc}
\hline Item set & Support count \\
\hline$\{1\}$ & 6 \\
$\{2\}$ & 7 \\
$\{3\}$ & 3 \\
$\{4\}$ & 8 \\
$\{5\}$ & 10 \\
$\{6\}$ & 12 \\
\hline
\end{tabular}

The one-dimensional maximum item set (L1) is generated, as shown in table 3 . The candidate item set $\mathrm{C} 2$ is generated by $\mathrm{L} 1$, such as $\{1,2\},\{1,3\},\{1,6\},\{2,3\},\{2,6\}$ and $\{3,6\}$.Scanning D, the correlation degree of each candidate item set is counted, the result is shown as table 4 . The two-dimensional maximum item set L2 is obtained by the same way, as shown in table 5 .

Correlation set $\mathrm{C} 3$ is generated from $\mathrm{L} 2$, as $\{1,2,3\}$. By comparing the candidate support count with the minimum support MinSupport, the three-dimensional maximum item set L3 is $\{1,2,3\}$, and support count is 6 . Therefore, through the training and recognition of the work order, the index mining processing is realized according to the index correlation degree. As shown in figure 6.

Table 3. One-dimensional maximum project set

\begin{tabular}{ll}
\hline Item set & Support count \\
\hline$\{1\}$ & 6 \\
$\{2\}$ & 7 \\
$\{3\}$ & 10 \\
$\{6\}$ & 12 \\
\hline
\end{tabular}

Table 4. One-dimensional maximum project set

\begin{tabular}{ll}
\hline Item set & Support count \\
\hline$\{1,2\}$ & 6 \\
$\{1,3\}$ & 7 \\
$\{1,6\}$ & 10 \\
$\{2,3\}$ & 12 \\
$\{2,6\}$ & - \\
$\{3,6\}$ & - \\
\hline
\end{tabular}

Table 5. One-dimensional maximum project set

\begin{tabular}{cc}
\hline Item set & Support count \\
\hline$\{1,2\}$ & 6 \\
$\{1,3\}$ & 7 \\
$\{1,6\}$ & 10 \\
$\{3,6\}$ & - \\
\hline
\end{tabular}




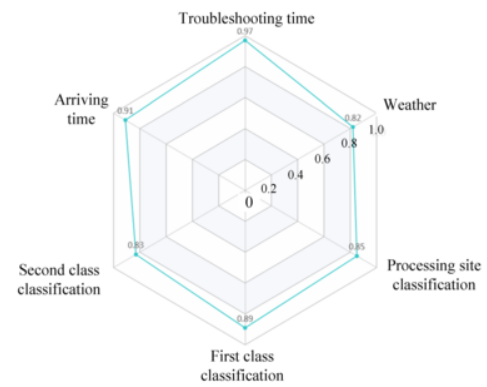

Figure 6. Analysis on key influencing factors of the average response time

\subsection{Quick Identification and Judgment}

The process of calculating and analysis is shown in figure 7.

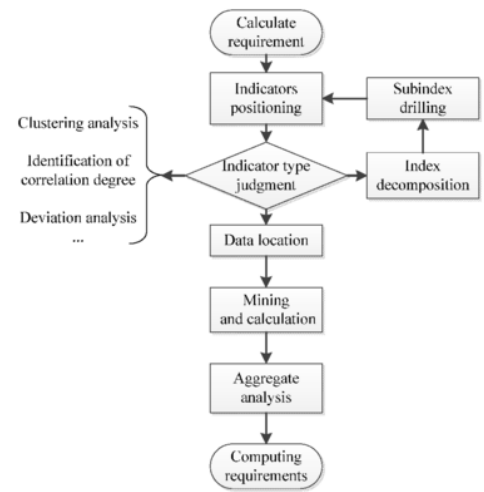

Figure 7. The process of the rapid identification and judgment of index traceability source data

The index of average return time is defined by multi-dimensional sinking algorithm, and then they are analyzed in the sub-nodes to obtain the demand index and to capture the index traceability process.

\section{Conclusion}

Based on the research of big data analysis technology, this paper makes a comprehensive analysis of the current distributed data architecture of the DN. The W-A DSCT was proposed to realize the visual construction of the algorithm, the active encapsulation of sinking and multiple iterations of calculation methods. The OCMS of the first-level management and analysis node and the second-level calculation of sinking node, which is suitable for the management of DN was built. Based on the principle of "moving computation, not moving to data", the provincial PSSC system and full-service data center/big data platform are fully connected to achieve dual-penetration of data and analysis. Deep mining and analysis of big data of DN can be realized based on machine learning algorithms such as cluster analysis and correlation analysis, so as to improve the lean management level of DN. 


\section{References}

[1] HOU Dan, LI Gang, ZHAO Wenqing, YANG Lu et al. 2015 Application of Big Data Analysis and Visualization Technology in Power Grid Company 3(12):1186-119(in Chinese).

[2] FAN Liping, ZHANG Xiaohui, Su Wei et al 2019 Research and application of substation equipment fault early warning based on big data mining technology 22(01):1-7 (in Chinese).

[3] Shi Changkai, Zhang Bo, Sheng Wanxing et al 2013 A discussion on technical architecture for flexible intelligent interactive power utilization[J]. Power System Technology 37(10):2868-2874(in Chinese).

[4] Xu Taishan, Xu Jianbing, Bao Yanhong et al 2006 Online predecision based preventive and emergency control system for interconnected power grids [J]. Automation of Electric Power Systems, 30(7):1-4(in Chinese).

[5] YANG Tao, HUANG Junkai, XU Kui et al 2018 Diagnosis method of power transformer fault based on deep learning [J]. Power Systems and Big Data 21(06): 23-30.

[6] ZHANG Nan, LI Zhi, LIU Di, LI Chunyang, HU Guanglin, YANG Zhanggui 2019 Research on Resource Management of Power Cloud System Based on Sinking Computation [J]. Power System Technology 43(12):4322-4328.

[7] Pradhan P, Behera P K, Ray B N B 2016 Modified round robin algorithm for resource allocation in cloud computing [J]. Procedia Computer Science 85:878-890.

[8] Huang Li,Wei Zhinong, Wei Yanfang et al 2013 A survey on interactive system and operation patterns of intelligent power utilization[J]. Power System Technology 37(8):2230-2237(in Chinese).

[9] LU Xiao, LIU Yi, JIANG Yefeng, Jing, FAN Qing, ZHANG Huacheng,et al 2019 Overall design of artificial intelligence application based on monitoring big data platform 22(04):37-42. (in Chinese).

[10] LI Zhanying 2017 Intelligent power distribution network and prospect analysis technology of data applications [J]. Power systems and big data 20(11): 18-20. 